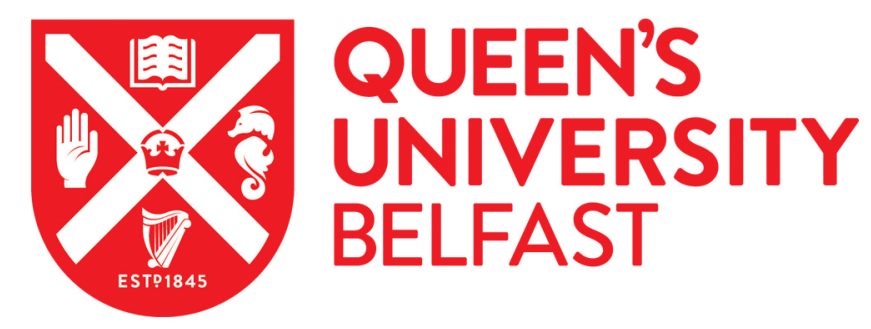

\title{
Super-Resolution Defect Characterization Using Microwave Near- Field Resonance Reflectometry and Cross-correlation Image Processing
}

Malyuskin, O., \& Fusco, V. (2017). Super-Resolution Defect Characterization Using Microwave Near-Field Resonance Reflectometry and Cross-correlation Image Processing. Sensing and Imaging, 18(7).

https://doi.org/10.1007/s11220-017-0157-8

Published in:

Sensing and Imaging

Document Version:

Peer reviewed version

Queen's University Belfast - Research Portal:

Link to publication record in Queen's University Belfast Research Portal

Publisher rights

(c) Springer Science \& Business Media New York 2017.

This work is made available online in accordance with the publisher's policies. Please refer to any applicable terms of use of the publisher

\section{General rights}

Copyright for the publications made accessible via the Queen's University Belfast Research Portal is retained by the author(s) and / or other copyright owners and it is a condition of accessing these publications that users recognise and abide by the legal requirements associated with these rights.

Take down policy

The Research Portal is Queen's institutional repository that provides access to Queen's research output. Every effort has been made to ensure that content in the Research Portal does not infringe any person's rights, or applicable UK laws. If you discover content in the

Research Portal that you believe breaches copyright or violates any law, please contact openaccess@qub.ac.uk. 


\title{
Super-Resolution Defect Characterization Using Microwave Near-Field Resonance Reflectometry and Cross-correlation Image Processing
}

\author{
Oleksandr Malyuskin ${ }^{1,2}$, Vincent Fusco ${ }^{1}$ \\ ${ }^{1}$ ECIT Institute, Queen's University Belfast, UK, BT3 9DT, ${ }^{2}$ School of Engineering, Ulster University Jordanstown, \\ UK. email: o.malyuskin@ulster.ac.uk
}

\begin{abstract}
A super-resolution defect characterization technique based on near-field resonance reflectometry and cross-correlation image processing is proposed in this paper. The hardware part of the microwave imaging system employs a novel loaded aperture (LA) probe which allows collimation of the electromagnetic field to approximately $\lambda / 10$ focal $\operatorname{spot}(\mathrm{s})$ at $\lambda / 100$ to $\lambda / 10$ stand-off distances, $\lambda$ being the wavelength of radiation in free space. The characteristic raw image spatial resolution of the LA probe is around $\lambda / 10$ in one dimension with amplitude contrast/sensitivity exceeding 10-20dB. It is demonstrated that the LA spatial resolution can be at least two times enhanced in two dimensions in the image plane using basic crosscorrelation image processing while retaining a very high level of amplitude contrast of at least $10 \mathrm{~dB}$.
\end{abstract}

Keywords: Near-field reflectometry, microwave high-resolution imaging, aperture, electromagnetic field enhancement, measurement, image, correlation.

\section{Introduction}

Quality control and quality assurance procedures are often based on the comparison of a structure under test with a "standard" test sample. This comparison can be accomplished using optical imaging systems with subsequent image processing, however in many situations optical assessment tools cannot be used due to high cost of equipment and complexity of image processing algorithms. Also, the tested structures can be painted or covered with opaque protective coatings which restrict the application of optical-based quality control methods.

Microwave and millimeter-wave reflectometry [1]- [3] is a proven and widely-accepted imaging tool that permits defect characterization at relatively low cost, with the possibility of operation in both laboratory and industrial environments. Previously, microwave reflectometry has been applied to defectoscopy problems such as detection and evaluation of corrosion under paint and composite laminates [4], [5], fatigue crack detection on metal and dielectric surfaces [6], [7], non-destructive quality assessment of composites [2], etc. The essential part of any reflectometry system responsible for electromagnetic (EM) interaction with the sample and reflected signal 
collection is a sensing probe. This can be apertureless [8], [9] or aperture-based [1], [8], [10]. Apertureless probes are commonly represented by open-ended coaxial structures. These probes generate very tight near field pattern with full-width at half-maximum (FWHM) less that $\lambda / 10$ at around $\lambda / 50-\lambda / 100$ standoff distance however electromagnetic coupling of these probes to the sampled material is extremely weak, considerably limiting the image resolution amplitude contrast to less than 1-2dB [8]. The other class of probes is represented by the openended waveguide structures or apertures in conductive screens [1], [10]- [13]. The spatial resolution of these probes is limited by the aperture size, which in case of an open ended waveguide probe is approximately $\lambda / 4 \times \lambda / 2$. As the size of the aperture decreases, the transmitted power reduces, proportionally to the inverse third power of the aperture radius, which considerably limits the imaging distance and reduces the signal-to-noise ratio. Again, nearfield coupling of the aperture probes to the sample is weak, resulting in low imaging contrast between the imaged feature(s) and background (typically 1-3dB).

To overcome the limitations of the existing near-field aperture-based microwave probes it has been proposed to employ small apertures in combination with resonance antennas or through the introduction of conductive inserts positioned inside the aperture [14]. Such arrangements [14] lead to high level of EM coupling to the material under test since the dipole insert is parallel to the test surface [15]. The dipole insert can be folded which results in subdiffraction spatial resolution in two dimensions, [16], [17]. Due to efficient EM coupling the imaging contrast (the contrast between the imaged features and/or the features and the background) can exceed 10-20dB in amplitude and 100 degrees in phase in typical imaging scenarios [14], [16], [17]. Even though other resonance near field reflectometry probes, very small $(\sim \lambda / 10)$ in all three dimensions have been proposed previously, e.g. a helix antenna [17], the loaded aperture (LA) probe has a number of advantages, including high EM energy localization in the near-field zone. This energy and field localization enables imaging operation with very low power levels (typically as low as $-60 \mathrm{dBm}$ ) or, conversely, allows to achieve highest possible signal-to-noise ratio for a given input power level. At the same time, due to the extended length dimension $(\sim \lambda / 4)$ of the insert as compared to its width, very high raw image resolution is only possible in the direction orthogonal to the LA length [17]. Additionally, ghost dual-peak image features appear as a result of dipole-mode near field coupling which can be considered as a disadvantage for image interpretation [16]- [18].

In this paper a fundamental possibility to further enhance the spatial resolution of resonance LA probes using image processing techniques based on cross-correlation [17], [19], [20] is demonstrated. It is shown that the application of this image processing to the raw images obtained with the LA probes enables at least two times spatial resolution enhancement (to the resolvable features size $\sim \lambda / 25$ ) in both dimensions in the image plane and also eliminates dual-peak features that are present in the raw image. The technique was first proposed in our previous paper [17] and extended here to the case of arbitrary defects of various shape, size and symmetry. This technique is believed to be applicable to a wide range of defect characterization problems, including surface and shallow subsurface diagnostics, and should enhance spatial resolution of any compact near field probe. In this paper consideration is limited to the important practical case of metal-dielectric structures used in printed circuit boards 
(PCB) or antenna reflect-arrays [21]. In the present work, the PCB technology allows one to produce test samples and "defect" boards with controllable defects, including size and symmetry deviation for which the performance of the LA reflectometry system is analyzed.

\section{Imaging Setup}

Fig.1 shows the one-port imaging setup geometry, Fig.1a) and the LA probe detailed view, Fig.1b).

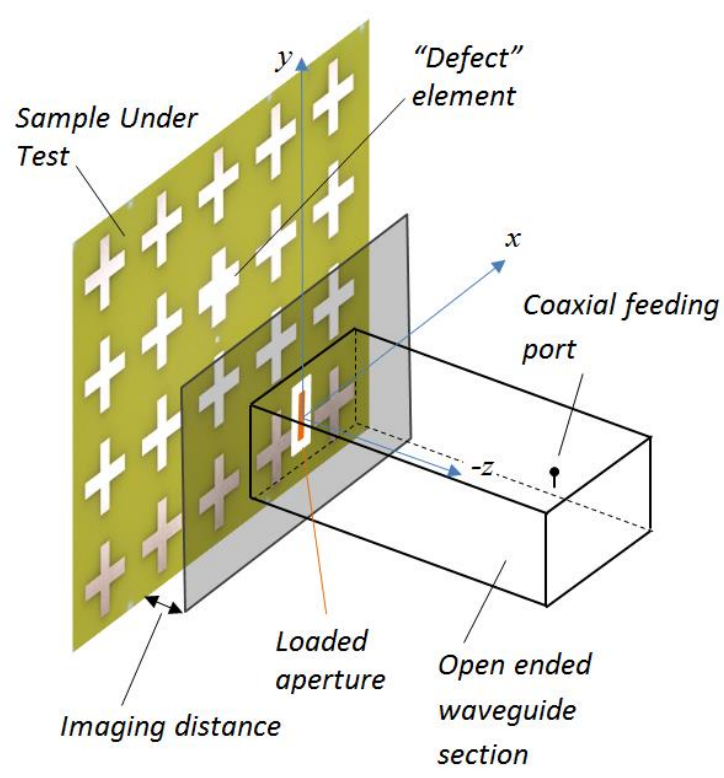

(a)

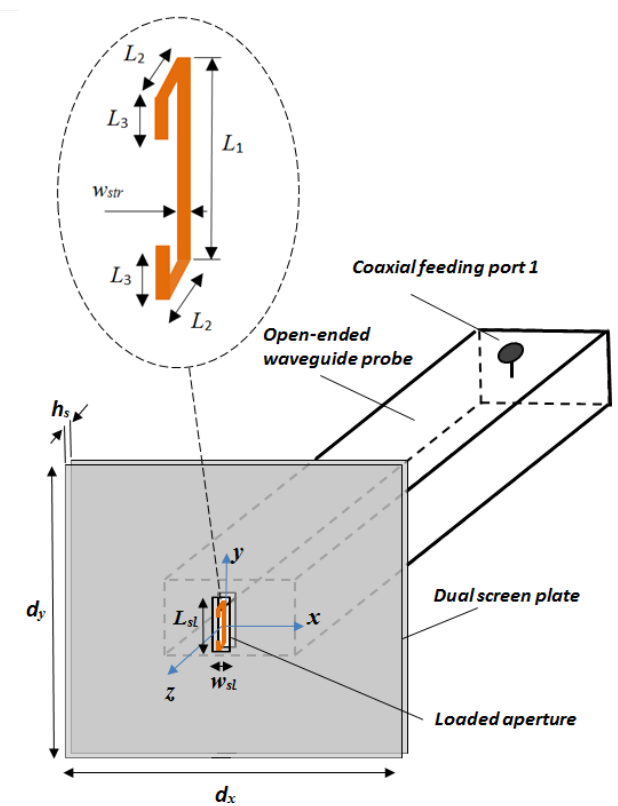

(b)

Fig.1.a) Imaging setup geometry; b) detailed view of the loaded aperture probe.

The LA probe is mounted on the planar near-field scanner with scan directions in the $x y$ plane. The sample is held in a vertical position on a wooden frame (not shown in Fig.1). As the probe scans the scene, varying nearfield probe-sample EM coupling is transformed into the reflection parameter $S_{11}$ amplitude and phase variation which forms a raw reflection image of the sample under test.

The geometrical parameters of the LA probe, Fig.1b) designed for $\sim 4 \mathrm{GHz}$ are summarized in Table 1.

TABLE 1. Dimensions (mm) of the LA probe

\begin{tabular}{lllllllll}
\hline \hline$d_{x}$ & $d_{y}$ & $h_{s}$ & $L_{s l}$ & $w_{s l}$ & $L_{1}$ & $L_{2}$ & $L_{3}$ & $w_{s t r}$ \\
\hline 66 & 66 & 3 & 25 & 7 & 18 & 3 & 6 & 2 \\
\hline \hline
\end{tabular}

The aperture is excited by the $\mathrm{TE}_{10}$ mode (with $E_{y}$ component of the EM field) propagating in the open-ended 
WR-187 waveguide section with flange dimensions $47.5 \times 22.1 \mathrm{~mm}$, which is fed by a coaxial port, Fig.1a).

The probe generates a dipole-mode transmitted near field pattern, Fig.2a) with the $E$-field significantly enhanced in the dipole-mode dual hot spots [14], [16], [17]. The probe supports very high-quality transmission resonance around $4.1 \mathrm{GHz}$, Fig.2b).

The near field focusing properties of the LA probe are shown to be in the subwavelength spatial range for imaging distance $\mathrm{z}<\lambda / 4$ with characteristic FWHM $\sim \lambda / 25$ at $\lambda / 70$ standoff distance and FWHM $\sim \lambda / 7$ at $\lambda / 10$ standoff distance. Experiments carried out in [14], [16], [17] demonstrate that in typical imaging scenarios involving dielectric materials and surfaces with conductive elements, the characteristic raw image resolution contrast (defined as variation of the $S_{11}$ parameter from feature to feature or from the feature to background) achievable with the LA probe exceeds $10-20 \mathrm{~dB}$ in amplitude and 100 degrees in phase of the reflection parameter $S_{11}$. High image resolution contrast arises as a result of spatial focusing and high-quality EM resonance of the LA probe. At the same time, the characteristic spatial resolution in the raw image is around $\lambda / 10$ in the $\mathrm{x}$ direction and around $\lambda / 4$ in the $y$ direction in the range of standoff distances $\lambda / 20-\lambda / 8$.

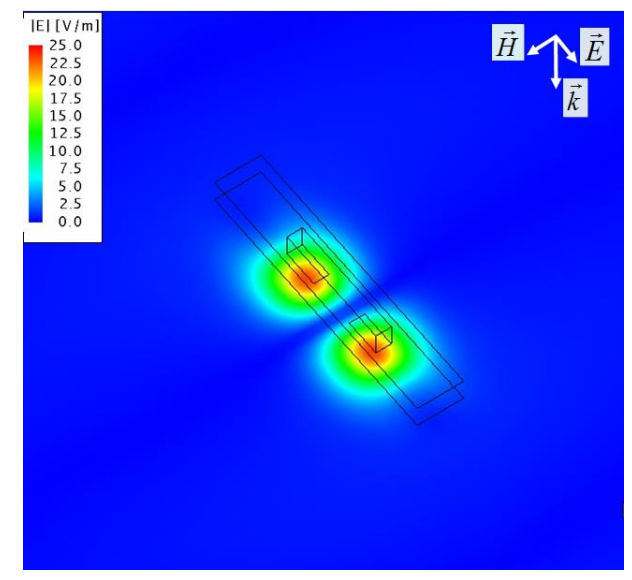

(a)

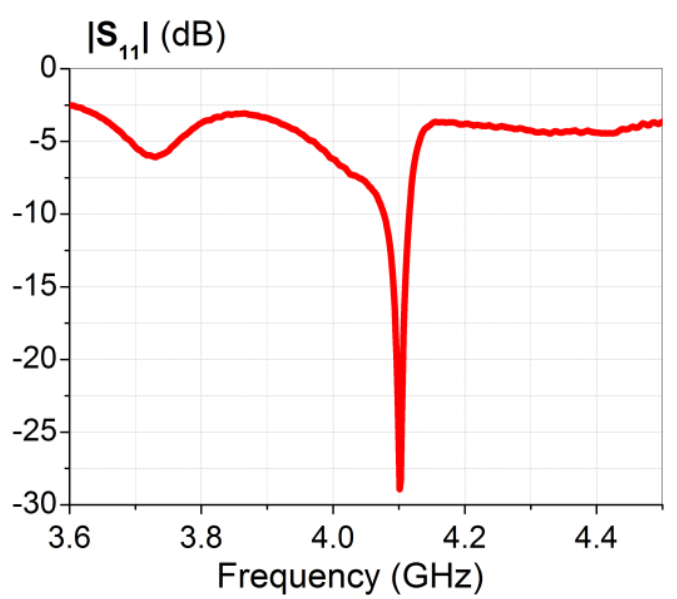

(b)

Fig.2.(a) Simulated near $E$-field amplitude distribution in the $x y$ plane at $z=4 \mathrm{~mm}(\lambda / 20)$; (b) measured $\mathrm{S}_{11}$ parameter magnitude.

\section{Mathematical Model of Defect Characterization in Periodic Printed Arrays Using Cross-Correlation}

Image correlation techniques can be used to enhance low-contrast features and detect minute changes in many imaging scenarios [17], [19], [20]. The cross-correlation technique for defect characterization is defined in this paper for comparison between the "master" flawless sample and the "defected" test structure, Fig.3. The technique is applicable to a general range of defectoscopy problems but is studied here only for a specific case of defect characterization in periodic printed antenna arrays. 


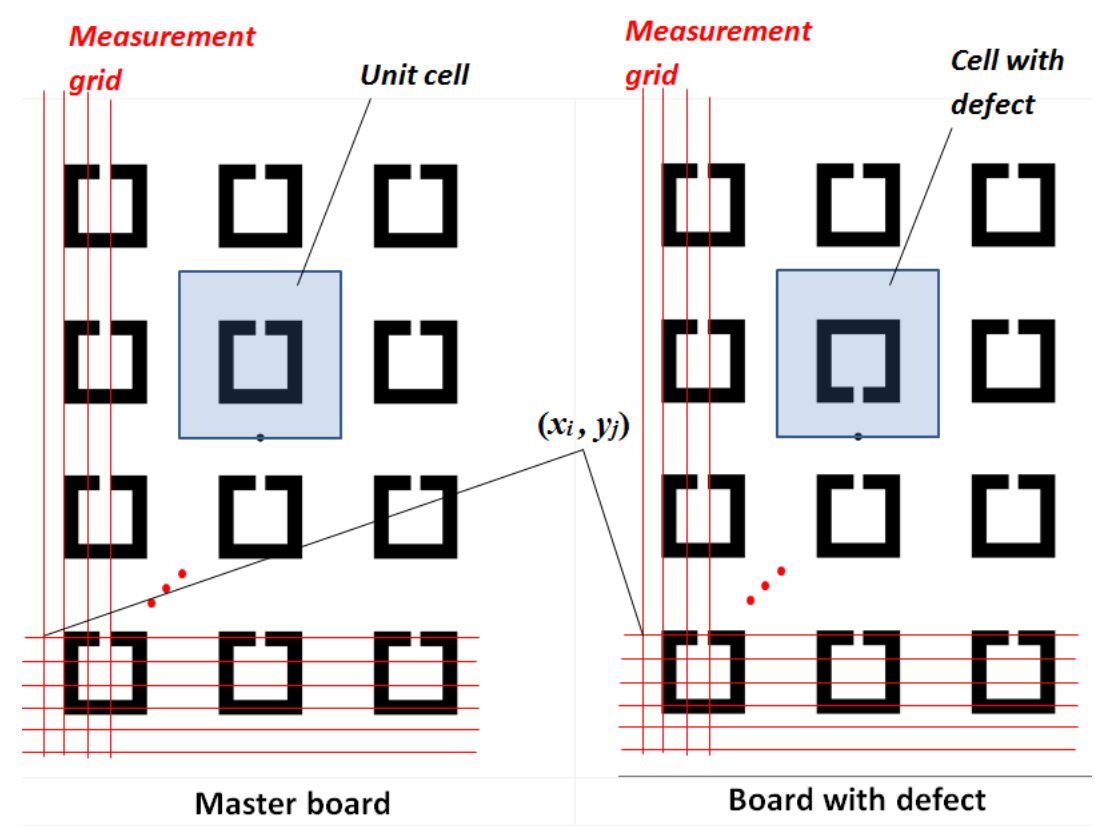

Fig.3. Correlation-based defect characterization in periodic printed antenna arrays

In the framework of this approach two $S_{11}$ measured data sets are compared: the first set of $S_{11}$ measured data belongs to the "master" board/periodic printed surface without defect and the second set of $\mathrm{S}_{11}$ measured data is obtained for the board with a "defect" element, Fig.3. The same measurement grids are used to obtain $\mathrm{S}_{11}$ data. It should be noted that instead of experimental data for the master board, simulation data can be used. The correlation-based processing technique is explained in detail below.

First, an auto-correlation function for the "master" board is calculated for each measurement point $\left(x_{i}, y_{j}\right)$,

$$
C_{M}\left(x_{i}, y_{j}\right)=R_{M} \cdot R_{M}^{*}
$$

In (1) $R_{M} \equiv R_{M}\left(x_{i}, y_{j}\right)$ is a complex-valued reflection coefficient for each measurement point $\left(x_{i}, y_{j}\right)$ for the "master" board, asterisk symbol denotes complex conjugate. Next the cross-correlation between the "master" and "defect" boards is computed for each measurement point as

$$
C\left(x_{i}, y_{j}\right)=R_{M} \cdot R_{D}^{*}
$$

where $R_{D} \equiv R_{D}\left(x_{i}, y_{j}\right)$ is a complex-valued reflection coefficient for each measurement point for the "defect" board, $i=1,2, . . N_{x}, j=1,2, . ., N_{y}$ where $N_{x}, N_{y}$ are the number of measurement points in $x, y$ directions respectively. 
The defect can be characterized by the normalized difference $f_{d}\left(x_{i}, y_{j}\right)$ of auto- and cross-correlation functions as

$$
f_{d}\left(x_{i}, y_{j}\right)=|\Delta C| / C_{\max }
$$

where $\Delta C \equiv C_{M}\left(x_{i}, y_{j}\right)-C\left(x_{i}, y_{j}\right)$, where $C_{\max }=\max \left[C_{M}, C_{D}\right]$ is a peak value of the autocorrelation function (1) or an autocorrelation function for the "defect" element $C_{D}\left(x_{i}, y_{j}\right)=R_{D} \cdot R_{D}^{*}$. The $f_{d}$ function can take any value in the range [0,1] with $f_{d} \rightarrow 1$ for strong defects with large spatial deviation from the "master" element and $f_{d} \rightarrow 0$ for the flawless sample. It should be noted that in (1)-(3) the defect characterization is achieved using a "local" or point-wise cross-correlation. This technique can be used also in the integral sense by summing (1), (2) over the area of interest, e.g. unit cell. To illustrate the proposed approach, various defects were considered and experimentally characterized, particularly those based on size and symmetry difference between the "master" and "defect" tests.

\section{Experimental Defect Characterization and Results Discussion}

PCB elements were printed on a $1.6 \mathrm{~mm}$ thick FR4 substrate without ground plane on the back side. The technique was applied for various standoff distances and measurement steps $\Delta x, \Delta y$. It has been observed that in the range of standoff distances $\lambda / 25-\lambda / 10$ the imaging resolution deteriorates proportionally to $z^{-1.5}$ where $z$ is an imaging distance [14], [16], [17]. It should be stressed that the measurement step defines the defect resolution and should be of order of the spatial defect deviation [17].

The set of possible defect elements representing typical situations when the defect varies in size, shape or symmetry is shown in Fig.4. The characteristic spatial defect deviation size is $\lambda / 20-\lambda / 25$.

Fig. 5 shows the printed array geometry, Fig. 5a) and cross-correlation function distribution, Fig. 5b) across the printed antenna array area $(300 \mathrm{~mm} \times 240 \mathrm{~mm})$. The array lattice dimensions are $52 \mathrm{~mm}$ x $52 \mathrm{~mm}$. The measurement steps are $\Delta x=3.5 \mathrm{~mm}, \Delta y=4.0 \mathrm{~mm}$. Imaging distance is $5 \mathrm{~mm}$ and frequency is $\sim 4 \mathrm{GHz}$. It can be seen that not only defect position can be clearly defined but also the geometry deviation area, encircled by red dotted circles in Fig.5a), is reconstructed. The dual-spot feature present in the near-field pattern of the probe, Fig.2a) is also eliminated. 


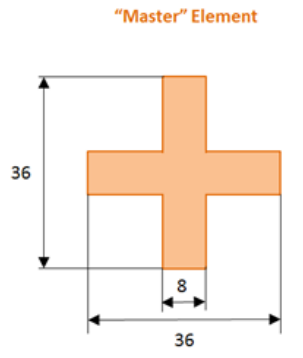

$$
\text { "Defect" Element }
$$
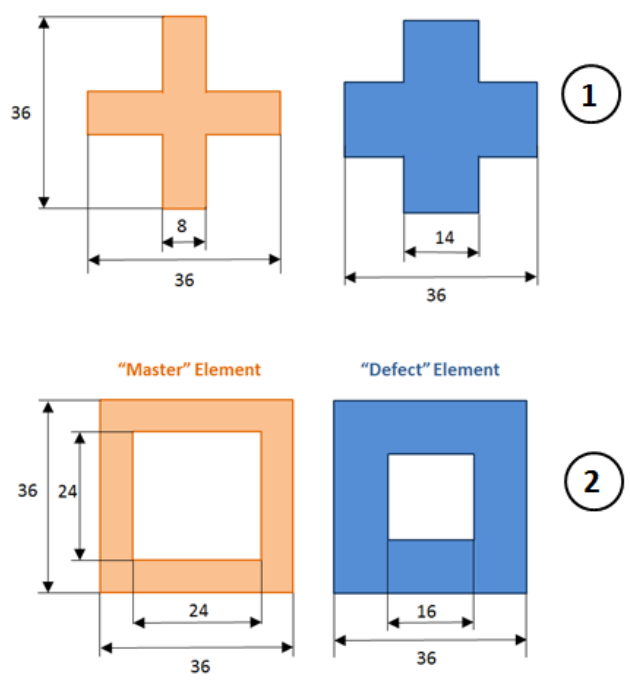

(2)

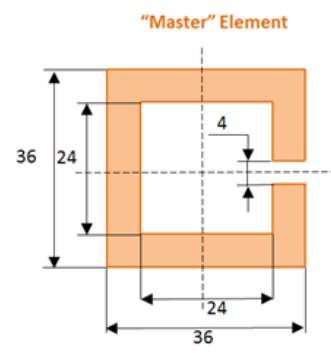

"Defect" Element

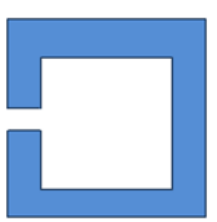

(3)

Fig.4. Set of "defect" elements used for the cross-correlation technique illustration. All dimensions in mm.

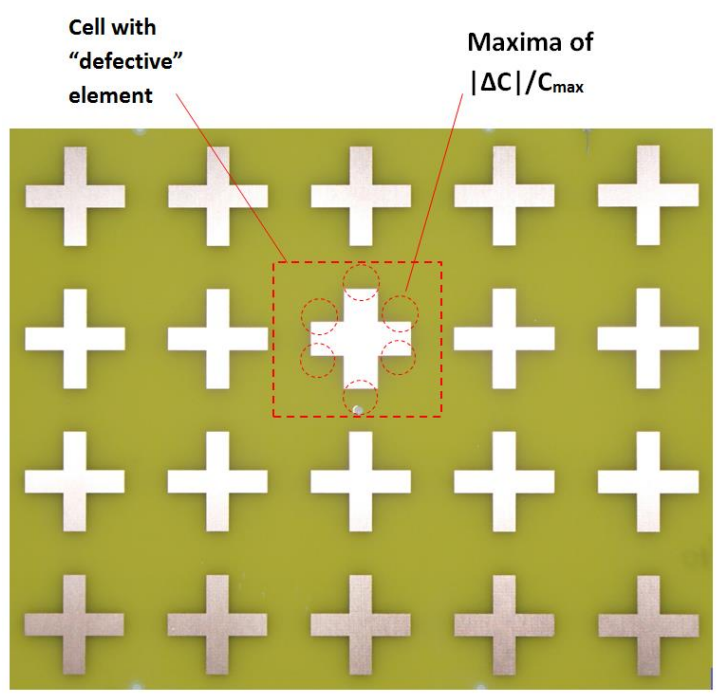

(a)

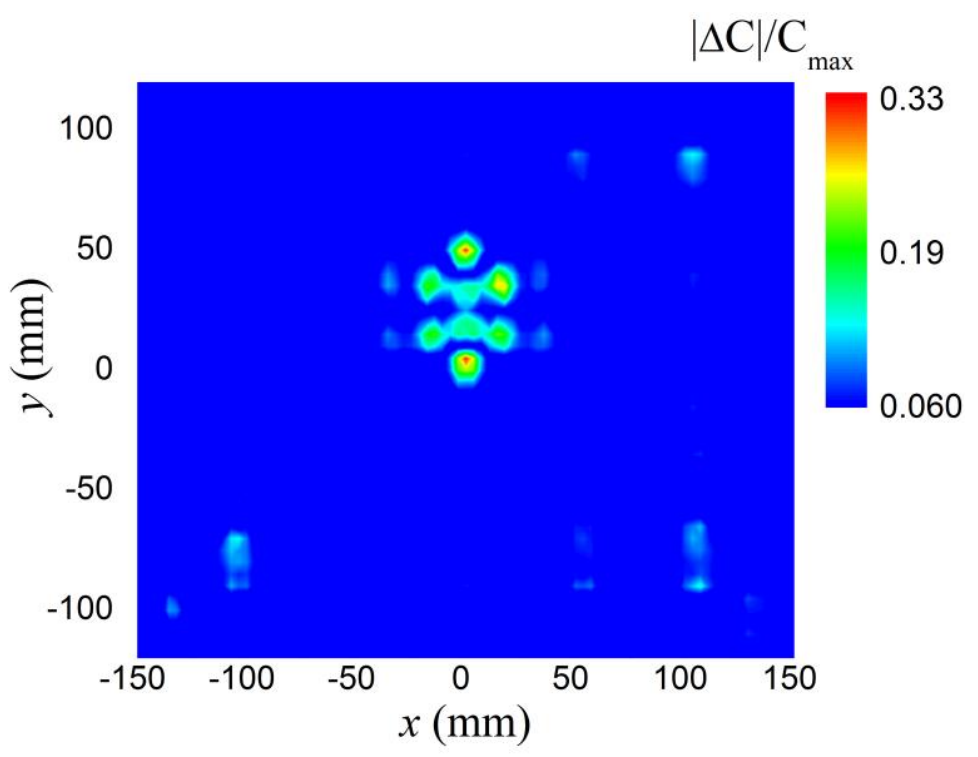

(b)

Fig.5. (a) Geometry of the board with defect type 1 and (b) cross-correlation function (3) distribution. In the top figure the cross-correlation function maxima corresponding to "defect" element are encircled for reference. 


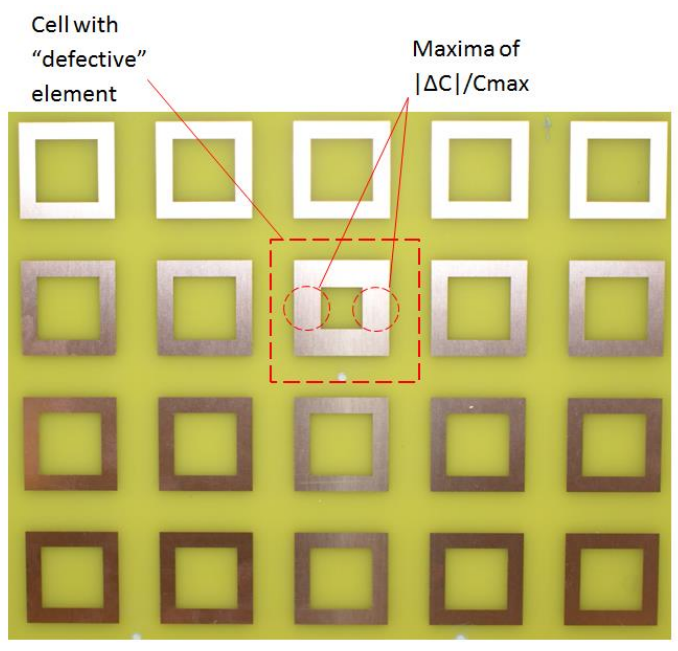

(a)

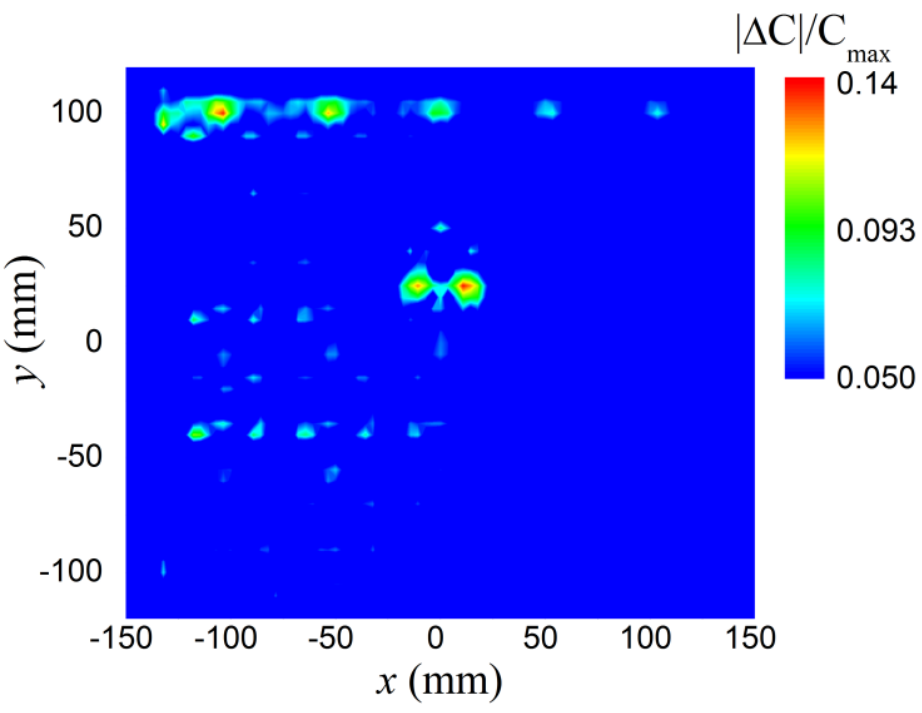

(b)

Fig.6. (a) Geometry of the board with defect type 2 and (b) cross-correlation function (3) distribution. In the top figure the cross-correlation function maxima corresponding to "defect" element are encircled for reference.

Fig.6a) shows the printed antenna array containing the "defect" element of type 2, and Fig.6b) shows the $f_{d}$ function distribution. The antenna array size, cell dimensions and imaging distance and measurement steps are the same as for the printed board in Fig.5a).

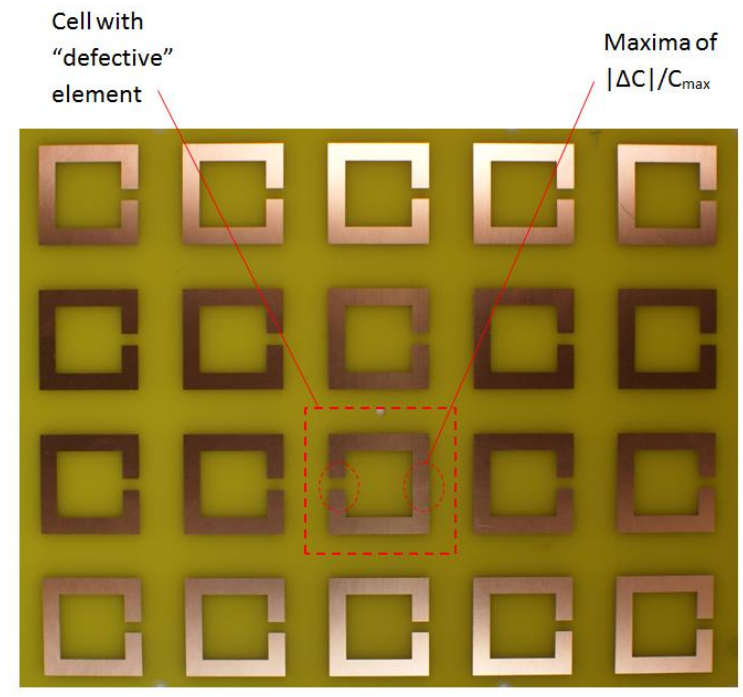

(a)

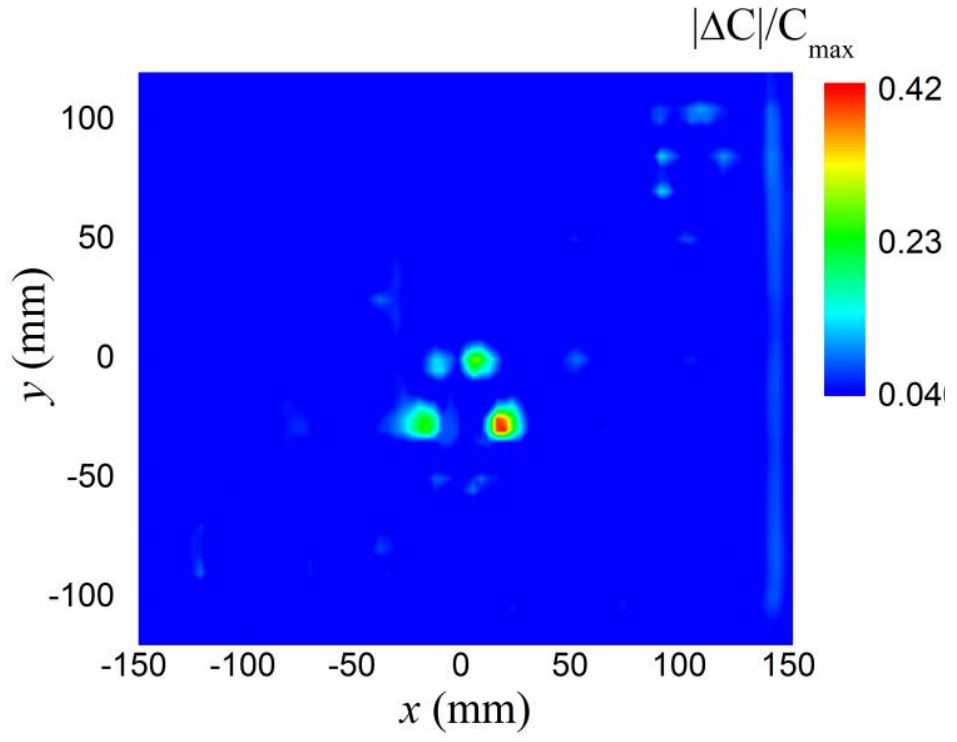

(b)

Fig.7. (a) Geometry of the board with defect type 3 and (b) cross-correlation function (3) distribution. 
It is interesting to note that not only the defect element is detected but also a high cross-correlation level is present for the top row of the printed elements located in the upper left corner of the board. This defect in the top left corner was caused by the "defect" PCB board bow detected by the microwave reflectometer. This defect is unlikely to be detected by an optical system with the optical axis aligned along the normal direction to the PCB board. In the case of the considered board with defect element of type 2 , the resolution contrast is $\sim 12 \mathrm{~dB}$ for the defect size $\sim \lambda / 20$.

Finally, Fig.7 demonstrates the printed array geometry (a) and cross-correlation function (b) distribution measured for the printed board with defect of type 3. It can be seen that the defect positions are clearly determined, Fig.7b)with relative contrast $21.4 \mathrm{~dB}$ to the average background level. Some asymmetry in the $f_{d}$ function distribution around the top and bottom parts of the "defect" broken loop is due do relatively coarse measurement step in $y$ direction.

Table 2.

Defect Characterization Based on Cross-Correlation Technique

\begin{tabular}{|c|c|c|c|}
\hline $\begin{array}{l}\text { Defe } \\
\text { ct } \\
\text { type }\end{array}$ & $\begin{array}{c}\text { Square } \\
\text { lattice } \\
\text { dimensions } \\
\quad(\mathrm{mm})\end{array}$ & $\operatorname{Max}\left(f_{d}\right)$ & $\begin{array}{c}\text { Average } \\
\left(f_{d}\right) \text { outside } \\
\text { defect cell }\end{array}$ \\
\hline \multirow[t]{2}{*}{1} & 52 & 0.33 & 0.005 \\
\hline & 68 & 0.30 & 0.006 \\
\hline \multirow[t]{2}{*}{2} & 52 & 0.14 & 0.009 \\
\hline & 68 & 0.17 & 0.006 \\
\hline \multirow[t]{2}{*}{3} & 52 & 0.42 & 0.003 \\
\hline & 68 & 0.40 & 0.005 \\
\hline
\end{tabular}

Table 2 summarizes the data for square lattice arrays with lattice (unit cell) dimensions $\mathrm{dx}=\mathrm{dy}=52 \mathrm{~mm}$, represented in Figs.5-7 and sparser arrays with unit cell dimensions $\mathrm{dx}=\mathrm{dy}=68 \mathrm{~mm}$. For example, for the defect of type 1 shown in Fig. 5a) the relative contrast between the peak of the cross-correlation difference function $f_{d}$ corresponding to encircled "defect" areas and the average background level of $f_{d}$ outside of the "defect" cell is

$$
10 \log \left(\max f_{d} / \bar{f}_{d \text { outside }}\right)=10 \log (0.33 / 0.005)=18.2 \mathrm{~dB}
$$


where $\bar{f}_{d}$ means average value. Therefore, the defects of characteristic size $\sim \lambda / 25$ can be very accurately characterized. It is interesting to note that for the bowed PCB in Fig.6 the average background level of $f_{d}$ outside of the "defect" cell is quite large, 0.009 , caused by the above mentioned board deformation.

From data in Table 2 it can be seen that the defects associated with each of the considered defect types, Fig. 4 can be very accurately detected and quantitatively characterized with relative contrast to background level better than $12 \mathrm{~dB}$. The experimental results obtained with finer imaging step $\Delta x=2.5 \mathrm{~mm}, \Delta y=4.0 \mathrm{~mm}$ show that in general further improvement of the resolution contrast by 1.8-2.5 $\mathrm{dB}$ is possible, this however requires longer acquisition times.

\section{Conclusions}

A high-resolution defect characterization technique based on the resonance focussed microwave reflectometry and image cross-correlation processing is proposed and experimentally evaluated in the case of printed antenna arrays. Experimental results demonstrate that deep subwavelength (characteristic size $\sim \lambda / 25$ ) geometry and symmetry two-dimensional defects can be robustly detected and quantitatively characterized. The defect resolution contrast is defined as the ratio between the maximum of the cross-correlation function corresponding to the defect position and the average level of the cross-correlation function outside the "defect" cell is shown to be from $12 \mathrm{~dB}$ to $20 \mathrm{~dB}$ for sub-wavelength defects of characteristic size $\lambda / 20-\lambda / 25$. The technique should find application in the broad range of surface and sub-surface imaging and defectoscopy scenarios.

\section{Acknowledgement}

This work was supported in part by the Leverhulme Trust, UK through the Grant RPG-2013-117. The authors would like to thank the anonymous reviewers for their insightful comments and suggestions that allowed them to improve the original manuscript.

\section{References}

[1] S. Kharkovsky and R. Zoughi, (2007) Microwave and millimeter wave nondestructive testing and evaluation , IEEE Instrum. Measurement Magazine, 26-37.

[2] J. Peebles, Non-Destructive testing of powders, ceramic, oils and other composite materials, MWI Laboratories Webcast, Dec. (2014) http://www.keysight.com/upload/cmc upload/All/11Dec14_MWIwebcast.pdf?\&cc=GB\&lc=eng

[3] S. Ivashov, A. Zhuravlev, M.Chizh and V. Razevig, "High resolution MW holographic system for NDT of dielectric materials and details", 16th Int. Conf. Ground Penetrating Radar, 2016, doi 10.1109/ICGPR.2016.7572595.

[4] S. Kharkovsky, A. Ryley, V. Stephen and Zoughi R.(2008) Dual-polarized near-field microwave reflectometer for noninvasive inspection of carbon fiber reinforced polymer-strengthened structures, IEEE Trans. Instrum. Meas.,57( 1), $168-175$. 
[5] S. Kharkovsky, J.T. Case, M.A. Abou-Khousa et.al (2006), Millimeter wave detection of localized anomalies in the Space Shuttle external fuel tank insulating foam, IEEE Trans. Instrum. Meas., 55(4), 1250-1257.

[6] M. Ghasr, B.J. Carroll , S. Kharkovsky et al.(2006) Millimeter wave differential probe for nondestructive detection of corrosion precursor pitting, IEEE Trans. Instrum. Meas., 55 (5), 1620-1627.

[7] J. Nadakuditi, G. Chen, R. Zoughi, (2006) Semiempirical electromagnetic modelling of crack detection and sizing in cement-based materials using near-field microwave methods IEEE Trans. Instrum. Measurements, 55(2), 588-597.

[8] B.T. Rosner B. T. and D. van der Weide.,(2002) High-frequency near-field microscopy, Review of Scientific Instruments, 73, 2505-2525.

[9] K. Haddadi and T. Lasri, (2013) Scanning microwave near-field microscope based on the multiport technology, IEEE Trans. Instrum. Measurement, 62(12), 3189 - 3193.

[10] A. Taeb , S. Gigoyan , S. Safavi-Naeini (2013), Millimetre-wave waveguide reflectometers for early detection of skin cancer, IET Microw. Antennas Propag., 7(14), 1182-1186.

[11]T. Pochiraju, O Malyuskin, V. Fusco, (2011) Tunable near- field sub-wavelength microwave imaging. Microwave and Optical Technology Letters, 53(6), 1229-1231.

[12]E.A. Ash, G. Nicholls, (1972) Super-resolution aperture scanning microscope, Nature, 237, 510-512.

[13] S. Dieter and W. Menzel , (2009) High-Resolution probes for near-field measurements of reflectarray antennas , IEEE Antennas Wireless Propagat. Lett., 8, 157-160.

[14] O. Malyuskin, V. Fusco, (2014) Near field enhancement and subwavelength imaging using resonantly loaded apertures, IEEE Trans Antennas Propagat, 62(6), 3130-3140.

[15] G. Smith, (1979) The electric-field probe near a material interface with application to the probing of fields in biological bodies , IEEE Trans. Microwave Theory Techniques, 27(3), 270-278.

[16] O. Malyuskin, V. Fusco (2015), Resonantly loaded apertures for sub-diffraction near-field imaging and surface probing, IET Microwaves, Antennas \& Propagation, 9(6), 533 - 540.

[17] O. Malyuskin, V. Fusco (2016), High-Resolution microwave near-field surface imaging using resonance probes, IEEE Trans. Instrumentation \& Measurement, 65(1), 189-200.

[18] O. Malyuskin, V. Fusco (2015), Resonantly loaded apertures for high resolution near-field surface imaging, IET Science, Measurement \& Technology, 9,783-791.

[19] M.A Sutton., J.-J. Orteu, H.W. Schreier, (2009) Image Correlation for Shape, Motion and Deformation Measurements, Springer, 2009.

[20] B. Pan (2011), Recent Progress in Digital Image Correlation, Experimental Mechanics, 51, 1223-1235.

[21] J. Huang, J.A.(2007) Encinar, Reflectarray Antennas, Wiley-IEEE.. 